University of Wollongong

Research Online

Faculty of Engineering and Information

Faculty of Engineering and Information

Sciences - Papers: Part A

Sciences

$1-1-2012$

Complementary techniques for probing terahertz magnetic excitations in Cu3Bi(SeO3)2O2Cl

\author{
$\mathrm{KH}$. Miller \\ University Of Florida \\ E Constable \\ University of Wollongong, evanc@uow.edu.au \\ H Berger \\ Institut de Physique Lausanne, Switzerland \\ D B. Tanner \\ University Of Florida \\ J Horvat \\ University of Wollongong, jhorvat@uow.edu.au
}

See next page for additional authors

Follow this and additional works at: https://ro.uow.edu.au/eispapers

Part of the Engineering Commons, and the Science and Technology Studies Commons

Research Online is the open access institutional repository for the University of Wollongong. For further information contact the UOW Library: research-pubs@uow.edu.au 


\title{
Complementary techniques for probing terahertz magnetic excitations in Cu3Bi(SeO3)2O2Cl
}

\author{
Abstract \\ A low frequency magnetic excitation in $\mathrm{Cu} 3 \mathrm{Bi}(\mathrm{SeO} 3) 2 \mathrm{O} 2 \mathrm{Cl}$ has been studied through complementary \\ spectroscopic techniques which utilised terahertz radiation from a synchrotron, a mercury arc lamp, and a \\ two-colour photomixing system. The excitation has been studied at temperatures down to $5 \mathrm{~K}$ and in \\ magnetic fields up to 10T. (C) 2012 IEEE. \\ Keywords \\ techniques, probing, 2o2cl, seo3, cu3bi, excitations, terahertz, magnetic, complementary \\ Disciplines \\ Engineering | Science and Technology Studies

\section{Publication Details} \\ Miller, K. H., Constable, E., Berger, H., Tanner, D. B., Horvat, J. \& Lewis, R. A. (2012). Complementary \\ techniques for probing terahertz magnetic excitations in $\mathrm{Cu} 3 \mathrm{Bi}(\mathrm{SeO}) 2 \mathrm{O} 2 \mathrm{Cl}$. International Conference on \\ Infrared, Millimeter, and Terahertz Waves, IRMMW-THz (pp. 1-2). Australia: IEEE.

\section{Authors} \\ K H. Miller, E Constable, H Berger, D B. Tanner, J Horvat, and R A. Lewis
}




\title{
Complementary techniques for probing terahertz magnetic excitations in $\mathrm{Cu}_{3} \mathrm{Bi}\left(\mathrm{SeO}_{3}\right)_{2} \mathrm{O}_{2} \mathrm{Cl}$
}

\author{
K. H. Miller, ${ }^{*}$ E. Constable, ${ }^{\dagger}$ H. Berger ${ }^{\ddagger}$ D. B. Tanner, ${ }^{*}$ J. Horvat ${ }^{\dagger}{ }^{\text {and R. A. Lewis }}{ }^{\dagger}$ \\ ${ }^{*}$ Department of Physics, University of Florida, Gainesville, Florida, USA

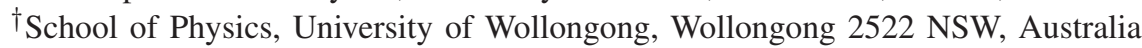 \\ $\ddagger$ Institute of Physics of Complex Matter, Ecole Polytechnique Federal de Lausanne, CH-1015 Lausanne, Switzerland
}

\begin{abstract}
A low frequency magnetic excitation in $\mathrm{Cu}_{3} \mathrm{Bi}\left(\mathrm{SeO}_{3}\right)_{2} \mathrm{O}_{2} \mathrm{Cl}$ has been studied through complementary spectroscopic techniques which utilised terahertz radiation from a synchrotron, a mercury arc lamp, and a two-colour photomixing system. The excitation has been studied at temperatures down to $5 \mathrm{~K}$ and in magnetic fields up to $10 \mathrm{~T}$.
\end{abstract}

\section{INTRODUCTION}

$\mathbf{S}$ INGLE crystals of the layered orthorhombic $\mathrm{Cu}_{3} \mathrm{Bi}\left(\mathrm{SeO}_{3}\right)_{2} \mathrm{O}_{2} \mathrm{Cl}$ compound were grown by the standard chemical vapor phase method. Below $24 \mathrm{~K}$, a long range magnetic order develops where the copper ions exhibit ferromagnetic intralayer and antiferromagnetic interlayer interactions. The $c$ axis, which is also the layering axis, is the preferred axis of magnetisation. A magnetic field of $0.7 \mathrm{~T}$ oriented along the $c$ axis triggers a metamagnetic transition where copper moments on every second layer flip, resulting in an overall ferromagnetic ordering in which the $c$ axis remains the preferred axis of magnetisation. More details of the magnetic properties as well as the temperature-dependent X-ray diffraction and infrared spectra of $\mathrm{Cu}_{3} \mathrm{Bi}\left(\mathrm{SeO}_{3}\right)_{2} \mathrm{O}_{2} \mathrm{Cl}$ are available elsewhere [1].

$\mathrm{Cu}_{3} \mathrm{Bi}\left(\mathrm{SeO}_{3}\right)_{2} \mathrm{O}_{2} \mathrm{Cl}$ transmits at frequencies below the strong infrared active phonon absorptions of the material (below $\sim 1.2 \mathrm{THz}$ or $40 \mathrm{~cm}^{-1}$ ). To obtain transmitted intensities in this region, complementary spectroscopic techniques were implemented that utilised three separate sources of terahertz (THz) radiation (a synchrotron, a mercury arc lamp, and a two-colour photomixing system). Spectra were obtained using synchrotron radiation in the frequency interval 0.45 $1.2 \mathrm{THz}\left(15-40 \mathrm{~cm}^{-1}\right)$ at the National Synchrotron Light Source, Brookhaven National Laboratory. The synchrotron radiation was modulated with a Bruker IFS 66-v/S spectrometer and subsequently transmitted through single crystals of $\mathrm{Cu}_{3} \mathrm{Bi}\left(\mathrm{SeO}_{3}\right)_{2} \mathrm{O}_{2} \mathrm{Cl}$ that were placed in a $10 \mathrm{~T}$ Oxford superconducting magnet. A similar Fourier transform infrared spectroscopy (FTIR) experiment was carried out utilising a mercury arc lamp in conjunction with a Polytec Michelson interferometer and a $7 \mathrm{~T}$ Oxford split-ring superconducting magnet in the spectral range $0.3-0.45 \mathrm{THz}\left(10-15 \mathrm{~cm}^{-1}\right)$ at the University of Wollongong. In addition, preliminary spectra were obtained using a two-colour photomixing system integrated into the existing optics of the Polytec spectrometer allowing the use of the same $7 \mathrm{~T}$ Oxford superconducting magnet mentioned above. The two-colour system has a frequency range of $0.06-1.2 \mathrm{THz}\left(2-40 \mathrm{~cm}^{-1}\right)$ and a spectral resolution of $\sim 100 \mathrm{Mz}\left(0.03 \mathrm{~cm}^{-1}\right)$. In all experiments transmitted intensities were collected with $4.2 \mathrm{~K}$ silicon bolometer detectors and absorption coefficients were subsequently calculated using a modified Beer-Lambert law that accounted for reflection losses at the surfaces.

The FTIR techniques using radiation from a synchrotron and a mercury arc lamp are both well established methods for magneto-optical experiments in the $\mathrm{THz}$ range. However, the coupling of a two-colour photomixing system to a superconducting magnet is a novel and advantageous technique not only for its spectral resolution and low frequency limitations, but also because the specific experimental setup mandated a portable source of $\mathrm{THz}$ radiation. Therefore, we proceed to describe the details of the experimental setup for two-colour photomixing incorporating a superconducting magnet, which are depicted in Fig.1a.

The two-colour photomixing $\mathrm{THz}$ system consists of a custom-made gallium-arsenide/low-temperature-grown gallium-arsenide (GaAs/LTG GaAs) photomixer, fabricated at the Technical University of Darmstadt. Terahertz radiation is produced by the well documented photoconductive heterodyne technique [4] using two frequency-offset near-infrared (NIR) diode lasers (Toptica DL-100, $\lambda_{0}=853 \pm 3 \mathrm{~nm}$ ) and a modulating bias of $0-9 \mathrm{~V}$ at $183 \mathrm{~Hz}$. The lasers are coupled to the photomixer using a polarisation maintaining optical fibre. The entire photomixer device itself is $\sim 50 \times 50 \mathrm{~mm}^{2}$. A continuous wave of monochromatic $\mathrm{THz}$ radiation is produced, which can be tuned by adjusting the frequency offset of the two NIR lasers. The small size and fibre coupling of the photomixer allows for a portable, tethered, continuous wave $\mathrm{THz}$ source. In this experiment we exploit the portability of the photomixer by integrating it into an existing magneto-optical setup. A $24 \mathrm{~m}$ polarisation maintaing optic fibre, optimised for $850 \mathrm{~nm}$ radiation, carrying the mixed NIR laser light, allows for precise positioning of the photomixer in different experimental setups around the laboratory with less than $10 \%$ loss of optical power. In the magnet setup the optical fibre is fed through an evacuation port into the spectrometer. The photomixer is fixed in place with an appropriate adjustable mount and the $\mathrm{THz}$ radiation it produces is collimated by a removable gold-plated off-axis parabolic mirror. The removable mirror allows for easy switching between the mercury arc lamp and photomixer 
$\mathrm{THz}$ sources.

\section{Results AND Discussion}

At $5 \mathrm{~K}$ and in an external field of $1 \mathrm{~T}$ applied parallel to the $c$ axis, a magnetic excitation is observed at $0.315 \mathrm{THz}$ $\left(10.5 \mathrm{~cm}^{-1}\right)$. As the field is increased, the excitation moves linearly to higher frequencies at a rate of $0.0303 \mathrm{THz}(1.01$ $\mathrm{cm}^{-1}$ ) per tesla. The resonance frequency as a function of magnetic field strength and corresponding absorption coefficients at all measured fields are shown respectively in Fig.1b and Fig.1c. The resonance was studied between 6 and $10 \mathrm{~T}$ using a synchrotron as the source of $\mathrm{THz}$ radiation. For fields less than $6 \mathrm{~T}$ the resonance moved below the measurable frequency range (below $0.45 \mathrm{THz}$ or $15 \mathrm{~cm}^{-1}$ ) of the particular experiment, a limit which was set by the optical filters used. The resonance was further studied between 1 and $5 \mathrm{~T}$ using the mercury arc lamp as the source of $\mathrm{THz}$ radiation, but for fields less than $1 \mathrm{~T}$ the resonance moved below the measurable frequency range of that particular experimental setup as well. The absorption coefficients measured using the synchrotron and the mercury arc lamp are shown in the right and left panels of Fig.1c respectively. Preliminary spectra using the two-colour photomixing system as a source were also recorded between 3.5 and $5.5 \mathrm{~T}$, and the results corroborate nicely with the previous data.

At $0.7 \mathrm{~T}$ a metamagnetic transition is triggered in which the magnetic interactions along the $c$ axis switch from antiferromagnetic below to ferromagnetic above the transition. The low frequency limitation and high resolution of the twocolour photomixing source make it an ideal tool to examine the resonance as it moves below $1 \mathrm{~T}$ and across the metamagnetic transition. Presumably one would expect an additional resonance branch arising below $0.7 \mathrm{~T}$ owing to existence of a second sublattice in the antiferromagnetically ordered state [2]. Further measurements below $1 \mathrm{~T}$ using the twocolour photomixing system are anticipated to investigate this possibility.

Polarisation dependent measurements of the excitation were carried out between 6 and $10 \mathrm{~T}$. The excitation was observed at all measured polarizations; namely, with the electric field of the light oriented along the $a$ axis, $b$ axis, and at $45^{\circ}$ to both the $a$ and $b$ axes. Further conclusions stemming from the polarisation dependence of the resonance cannot be drawn until it is studied at $0 \mathrm{~T}$.

The strength of the observed excitation corresponds to an optical conductivity of $0.23 \Omega^{-1} \mathrm{~cm}^{-1}\left(\sigma_{1}=\frac{c}{4 \pi} n \alpha\right)$, which is a typical strength of a single magnon excitation [1]. We therefore proceed to analyse the excitation as a magnon resonance. Kittel [3] determined the frequency dependence of a ferromagnetic magnon resonance when a magnetic field is applied parallel to the preferred axis to be $\hbar \omega=g \mu_{B} H_{\text {eff }}$. Where $g$ is the spectroscopic splitting factor and $H_{\text {eff }}$ is the effective magnetic field. Using the determined slope of the excitation between 1 and $10 \mathrm{~T}$ we arrive at a $g$ value of 2.16 , which is reasonable for a spin $\frac{1}{2}$ system (i.e., $\mathrm{Cu}^{2+}$ ).

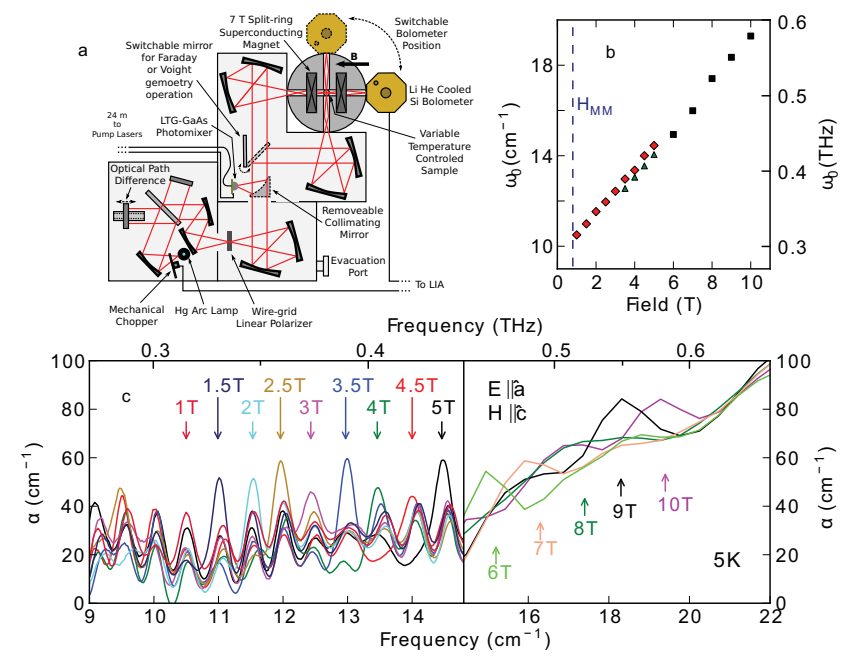

Fig. 1. The experimental setup (a) of the integrated two-colour photomixing system/Polytec spectrometer with superconducting magnet. The resonance frequency versus magnetic field (b) of the observed magnetic excitation in the interval 1-10 T. Data points obtained using a synchrotron as a source are plotted as black squares, mercury arc lamp are red diamonds, and the two-colour photomixing system are green triangles. $\mathrm{H}_{M M}$ denotes the field at which the metamagnetic transition occurs in the $\mathrm{H} \| c$ orientation. The absorption coefficients of the magnetic excitation at fields between 1 and $5 \mathrm{~T}$ using a mercury arc lamp (c left panel) and between 6 and $10 \mathrm{~T}$ using a synchrotron (c right panel). In the measurements shown the electric field of the light was oriented parallel to the $a$ axis.

\section{CONCLUSION}

Terahertz radiation from a synchrotron, a mercury arc lamp, and a two-colour photomixing system have been utilised to study the field dependence of a low frequency magnetic excitation in $\mathrm{Cu}_{3} \mathrm{Bi}\left(\mathrm{SeO}_{3}\right)_{2} \mathrm{O}_{2} \mathrm{Cl}$ at $5 \mathrm{~K}$. Further measurements using the two-colour photomixing system, which has emerged as an ideal system for low temperature and high field measurements below the lower frequency limits of conventional FTIRs, are needed.

\section{ACKNOWLEDGMENT}

K. H. M. thanks support from the NSF's EAPSI program, and the US DOE through contract DE-FG02-02ER45984 at UF and DE-AC02-98CH10886 at the NSLS. This work was supported by the Australian Research Council and by the University of Wollongong. We also thank Professor H. Hartnagel of the Technical University of Darmstadt for provision of the photomixer used in this work.

\section{REFERENCES}

[1] K. H. Miller et al., "Infrared phonon anomaly and magnetic excitations in single-crystal $\mathrm{Cu}_{3} \mathrm{Bi}\left(\mathrm{SeO}_{3}\right)_{2} \mathrm{O}_{2} \mathrm{Cl}$ ", unpublished.

[2] F. Keffer and C. Kittel, "Theory of Antiferromagnetic Resonance", Phys. Rev. 85, 329, 1952.

[3] C. Kittel, "On the Gyromagnetic Ratio and Spectroscopic Splitting Factor of Ferromagnetic Substances", Phys. Rev. 76, 743, 1949.

[4] E. R. Brown, K. A. McIntosh, K. B. Nichols and C. L. Dennis, "Photomixing up to $3.8 \mathrm{THz}$ in low-temperature-grown GaAs', Appl. Phys. Lett. 66 (3), 285, 1994. 